\title{
Evaluation of Positron Implantation Profiles in Various Materials for ${ }^{22} \mathrm{Na}$ Source
}

\author{
L.Yu. Dubov ${ }^{a, b, *}$, Yu.A. Akmalova ${ }^{a}$, S.V. Stepanov ${ }^{a, b}$, Yu.V. Funtikov ${ }^{b}$ \\ AND YU.V. SHTOTSKY ${ }^{a, b}$ \\ ${ }^{a}$ National Research Nuclear University MEPhI, 115409, Kashirskoe shosse, 31, Moscow, Russia \\ ${ }^{b}$ NRC Kurchatov Institute, Institute of Theoretical and Experimental Physics, \\ 117218, Bolshaya Cheremushkinskaya Str., 25, Moscow, Russia
}

\begin{abstract}
Positron implantation profiles in various materials for ${ }^{22} \mathrm{Na}$ isotope source are discussed. The spatial distribution of positrons in materials with densities from 1 to $21 \mathrm{~g} / \mathrm{cm}^{3}$ are simulated using GEANT4 code. The results indicate that depth distributions of thermalized positrons in any homogeneous medium can be described by the sum of two exponential functions. Contribution of the short-range exponent is about $11 \%$ in the materials. The both absorption coefficients can be well represented as a function of material density and atomic number. Proposed empirical model agrees well with available experimental data.
\end{abstract}

DOI: 10.12693/APhysPolA.132.1482

PACS/topics: 02.70.Uu, 29.90.+r

\section{Introduction}

Many problems related to diagnostics of near-surface layers can be solved by positron annihilation spectroscopy. Such tasks are typical for studies of irradiated samples, materials under mechanical loads and under influence of aggressive media, composite materials, coatings etc. Investigation of near-surface regions of matter using positrons emitted by isotope sources is limited by a large penetration depth of positrons in the samples. Therefore, slow monoenergetic positron beams are commonly used for measuring positron lifetime in thin nearsurface regions. However, these experiments are more complicated and less available than conventional isotopebased positron lifetime measurements.

Precise information about positron implantation profiles allows studying of nonuniform defects distribution using radioisotope $e^{+}$sources and reconstructing in some cases concentration of positron trapping sites in the nearsurface region.

Distribution of annihilation events vs. $e^{+}$penetration depth in the sample practically coincides with the distribution of the points where $e^{+}$become thermalized, because the diffusion length of thermalized $e^{+}$is negligible in comparison with the range of fast positrons ejected by the isotope sources. The probability density function (pdf) of the thermalized $e^{+}$(positron implantation profile) is usually considered to be monoexponential function

$$
f(x)=\mathrm{d} P(x) / \mathrm{d} x=\alpha \mathrm{e}^{-\alpha x},
$$

where $\mathrm{d} P(x)$ is the probability of positron slowing down to thermal energies at the depth between $x$ and $x+\mathrm{d} x$, $\alpha$ is a linear absorption coefficient, which can be calculated, for example, by the formula proposed by Dryzek

\footnotetext{
*corresponding author; e-mail: LYDubov@mephi.ru
}

and Singleton [1]:

$$
\alpha=12.6 \rho Z^{0.17} / E_{\max }^{1.28}\left[\mathrm{~cm}^{-1}\right],
$$

where $\rho$ is the material density in $\mathrm{g} / \mathrm{cm}^{3}, Z$ is the average atomic number of material, and $E_{\max }$ is the maximal energy of $\beta^{+}$-spectrum in $\mathrm{MeV}$. For ${ }^{22} \mathrm{Na}$ source $E_{\max }=0.545 \mathrm{MeV}$.

More sophisticated analytical descriptions of the positron depth distribution were suggested in [2-4]. In our paper we present the results of the positron implantation profile simulations basing on GEANT4 code for a number of substances with different densities from 1 to $21 \mathrm{~g} / \mathrm{cm}^{3}$ and propose analytical expression for such descriptions.

\section{Simulated physical processes}

We study the passage of positrons through matter in the source-sample "sandwich" geometry, when ${ }^{22} \mathrm{Na}$ source is arranged in between two investigated samples. The positron source consists of $\mathrm{NaCl}$ layer placed between two kapton films. The source ejects positrons isotropically within $4 \pi$ solid angle. Then positrons experience multiple scattering in kapton envelop and in the samples. During thermalization process positrons may repeatedly pass from one material to another.

A positron, passing through a matter, loses its energy mainly due to inelastic collisions with atomic electrons. Energy losses includes continuous losses (excitation of atoms and production of low energy ions) and discrete losses related to formation of the high energy secondary electrons.

To simulate tracks of charged particles in GEANT4 a multiple scattering model based on the Lewis multiple scattering theory is used. Energy losses at each step are calculated by the Bethe-Bloch formula, and after each step the bias and the new direction are calculated using randomization.

An alternative to the multiple scattering process is the single elastic scattering model. It is more accurate, 
but requires much longer computation time in comparison with the multiple scattering model. In our calculations, the single scattering processes are used to describe positron interactions in close vicinity of material boundaries, when the trajectory step size in the multiple scattering model exceeds the distance to the boundary. This approach allows to avoid errors when positron crosses boundaries from one material to another. To describe positron interactions within studied materials we used multiple and single scattering process from the library of electromagnetic processes PENELOPE (PENetration and Energy LOss of Positrons and Electrons) included in GEANT4, which had been developed for the most accurate Monte Carlo simulation of low-energy interactions of light charged particles.

\section{Simulation of the positron implantation profile}

The geometrical model and the description of physical processes was developed in the GATE/GEANT4 environment [5]. The positron source consists of a layer of radioactive salt $\mathrm{NaCl}$ of $\approx 1 \mu \mathrm{m}$ thickness and two kapton films $\left(2 \times 8 \mu \mathrm{m}^{2}\right)$. Materials with different densities (from water to uranium) are used as samples.

Before entering the sample, positrons pass through the kapton films of the source. Part of positrons annihilates therein. Others lose there part of their kinetic energy. Some positrons are backscattered in the samples and increase the source contribution in the observed annihilation spectrum. Table I shows source contributions calculated for the materials discussed below. The obtained results are in a good agreement with our experimental data and the calculations reported in [6] with a correction for the thickness of the kapton layer and presence of $\mathrm{NaCl}$ salt.

The energy spectrum of positrons emitted by the ${ }^{22} \mathrm{Na}$ radionuclide and the spectrum of positrons entering the samples simulated in GEANT4 are shown in Fig. 1. The spectrum of the positrons entering the sample is shifted to lower energies. The magnitude of this effect depends mainly on the density and thickness of the source envelope and very weakly depends on the material of the sample.

Two positron implantation profiles for tungsten are shown in Fig. 2. Dashed line shows monoexponential calculations, Eq. (1) and Eq. (2). Dots show the result of our simulation in GEANT4. Solid line represent the two-exponential approximation of the simulated profile. We can see that Eq. (1) substantially underestimates positron density at depths less than $1 \mu \mathrm{m}$.

Simulated implantation profiles (probability density functions) of positrons for substances with a density from $1 \mathrm{~g} / \mathrm{cm}^{3}$ (water) to $19.3 \mathrm{~g} / \mathrm{cm}^{3}(\mathrm{Au})$ are shown in Fig. 3. The mean range of implanted positrons varies from a few $\mu \mathrm{m}$ for high density materials to several $\mathrm{mm}$ for low density.

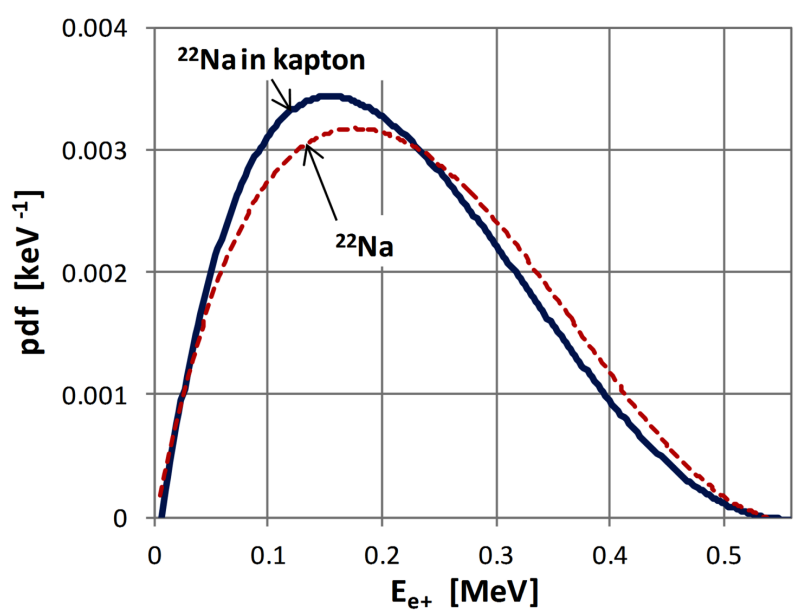

Fig. 1. Energy spectrum of the positrons emitted by ${ }^{22} \mathrm{Na}$ nuclei (dashed line), solid line - the same spectrum for the source placed in a $8 \mu \mathrm{m}$ kapton envelope.

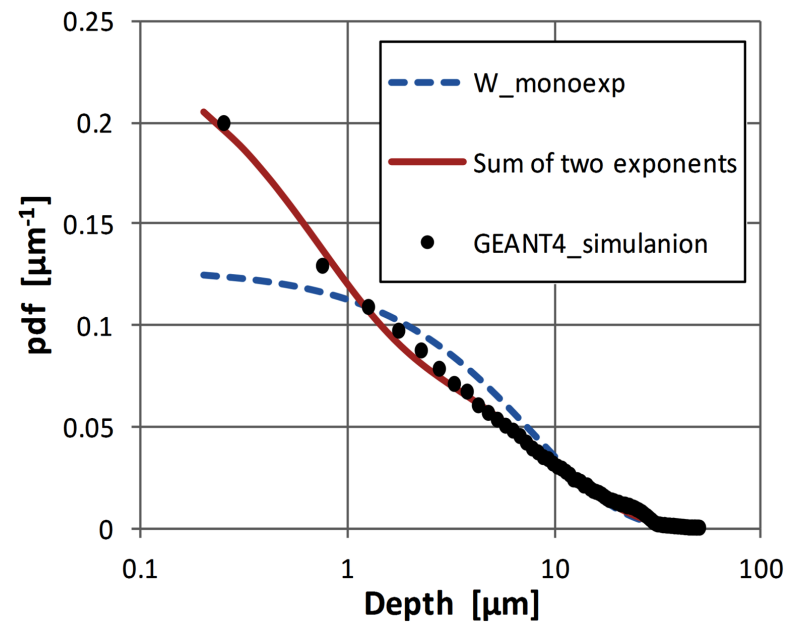

Fig. 2. Positron implantation profiles in tungsten.

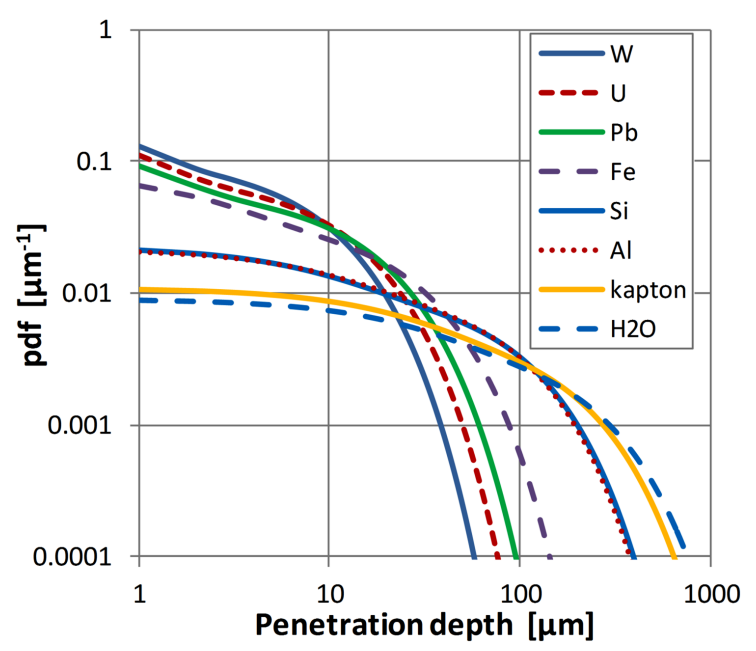

Fig. 3. Simulated $e^{+}$implantation profiles (probability density functions) for various materials. 
Source contributions (in \%) for various studied materials (samples).

TABLE I

\begin{tabular}{c|c|c|c|c|c|c|c|c|c|c|c|c}
\hline \hline $\mathrm{H}_{2} \mathrm{O}$ & Kapton & $\mathrm{Al}$ & $\mathrm{Si}$ & $\mathrm{Fe}$ & $\mathrm{Ge}$ & $\mathrm{Mo}$ & $\mathrm{Sn}$ & $\mathrm{W}$ & $\mathrm{Pt}$ & $\mathrm{Au}$ & $\mathrm{Hg}$ & $\mathrm{Pb}$ \\
\hline 12.3 & 12.2 & 13.3 & 14.3 & 16.2 & 17.3 & 18.2 & 19.7 & 21.1 & 21.3 & 21.4 & 21.4 & 21.5
\end{tabular}

Absorption coefficients $a$ and $b\left[\mu \mathrm{m}^{-1}\right]$ for simulated materials.

TABLE II

\begin{tabular}{c|c|c|c|c|c|c|c|c|c|c|c|c|c}
\hline \hline & $\mathrm{U}$ & $\mathrm{Pb}$ & $\mathrm{Hg}$ & $\mathrm{Au}$ & $\mathrm{Pt}$ & $\mathrm{W}$ & $\mathrm{Mo}$ & $\mathrm{Ge}$ & $\mathrm{Fe}$ & $\mathrm{Si}$ & $\mathrm{Al}$ & Kapton & water \\
\hline$a$ & 1.6 & 1.1 & 1.3 & 1.8 & 1.9 & 1.7 & 0.72 & 0.30 & 0.43 & 0.11 & 0.10 & 0.044 & 0.037 \\
$b$ & 0.10 & 0.067 & 0.078 & 0.11 & 0.13 & 0.11 & 0.056 & 0.028 & 0.042 & 0.012 & 0.013 & 0.0062 & 0.0050
\end{tabular}

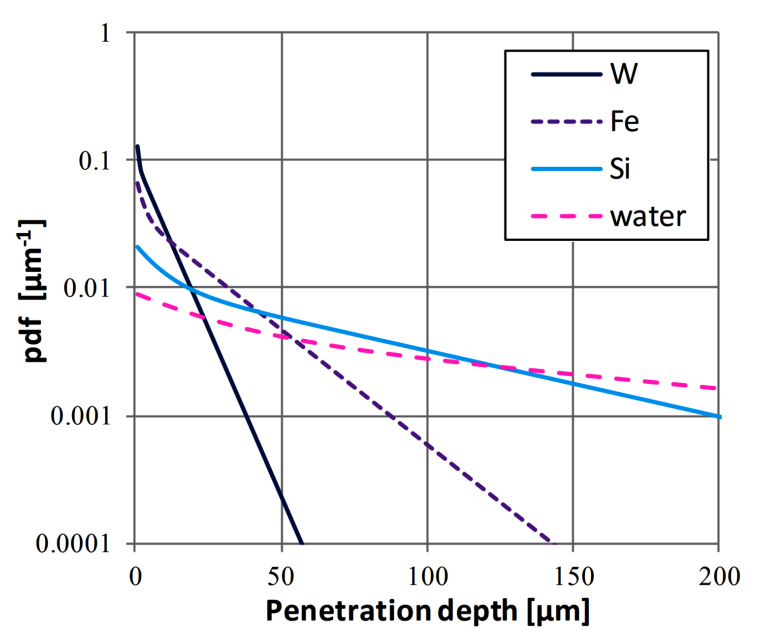

Fig. 4. Simulated $e^{+}$implantation profiles in tungsten, iron, silicon, and water in semilogarithmic scale.

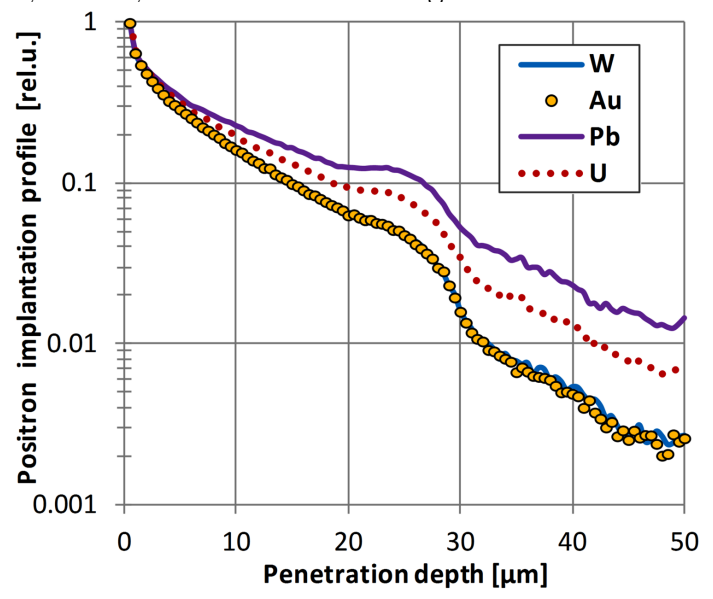

Fig. 5. Simulated $e^{+}$implantation profiles in heavy elements.

Figure 4 shows some implantation profiles in semilogarithmic scale. The presence of two different exponential components is clearly seen.

For low density materials positron implantation profiles are fairly smooth at all depths, but in high-density materials profiles have more complex shape. Figure 5 shows simulated profiles for heavy elements. A distinctive feature of these profiles is the presence of a bulge at $20-30 \mu \mathrm{m}$. It is apparently associated with the high energy positrons, which are injected into the sample perpendicular to its surface. The contribution of the bulge is no more than $1 \%$ and allows us to approximate the implantation profile using smooth functions.

\section{Approximation of the positron implantation profiles}

The simulated positron implantation profiles in various samples can be described with a good accuracy by the sum of two exponential distributions

$$
f(x)=\eta a \mathrm{e}^{-a x}+(1-\eta) b \mathrm{e}^{-b x}
$$

where $\eta$ is the contribution of the short-range component corresponding to the low energy positrons and positrons entering the sample at small angles to the surface. For all analyzed materials, contribution of the short-range component is approximately $\eta=11 \pm 1 \%$. Absorption coefficients $a$ and $b\left[\mu \mathrm{m}^{-1}\right]$, obtained by approximation of the simulated profiles with the sum of two exponents, are shown in Table II.

The absorption coefficient $a$ of the short-range component can be described by the formula

$$
a=135 \rho Z^{0.435}\left[\mathrm{~cm}^{-1}\right] .
$$

The coefficient $b$ of a long-range component only slightly differs from values given by Eq. (2). Minimizing deviations we obtained for $b$ the following expression:

$$
b=37.4 \rho Z^{0.1}\left[\mathrm{~cm}^{-1}\right] .
$$

These empirical equations allow to calculate absorption coefficients for all considered materials (except uranium) with good accuracy. For uranium $(Z=92)$, the discrepancy is more than $10 \%$.

\section{Experimental verification}

To confirm our simulations for kapton, we measured various set of kapton films placed in between the positron source and thick teflon wafers. Since the atomic numbers and the densities of kapton and teflon are close enough, backscattering from the samples does not introduce significant distortions into the distribution of positrons in thin layers of kapton. Thickness of the kapton films was varied from $8 \mu \mathrm{m}$ to $150 \mu \mathrm{m}$. Measured positron lifetime spectra were decomposed into into short $(<400 \mathrm{ps})$ and long ( $>1 \mathrm{~ns}$ ) exponential components. Since there is no positronium formation in kapton, the intensity of the long-lived component in the observed positron lifetime spectrum is proportional to the number of positrons that reach teflon wafers. Taking into account source contribution we determine the fraction of positrons annihilated in teflon (Fig. 6).

The normalized amount of positrons reached teflon wafers (the positron transmittance function) can be calculated from the $e^{+}$implantation profile in kapton $f(x)$ as follows: 


$$
I(z)=\int_{z}^{\infty} f(x) \mathrm{d} x .
$$

It is the fraction of positrons passed through the kapton layer of the thickness $z$. Figure 6 shows $I(z)$ calculated according to Eqs. (3) and (6) and our experimental results. For comparison we have also demonstrated $I(z)$ calculated by the monoexponential formula (Eq. (1)) and the two-component transmittance function

$$
I(z)=\left\{\begin{array}{ll}
\frac{N}{2}\left(\exp (-2 \alpha z)+\exp \left(-2 \alpha d_{0}\right)\right), & z \leq d_{0} \\
N \cdot \exp \left(-2 \alpha\left(z+d_{0}\right)\right), & z \leq d_{0}
\end{array},\right.
$$

suggested by Dryzek and Singleton [1]. Here $z$ is the penetration depth, $\alpha$ is the absorption coefficient given by Eq. (2), $N=2 /\left(1+\exp \left(-2 \alpha d_{0}\right)\right)$ is the normalization constant and $d_{0} \approx 0.5 / \alpha$.

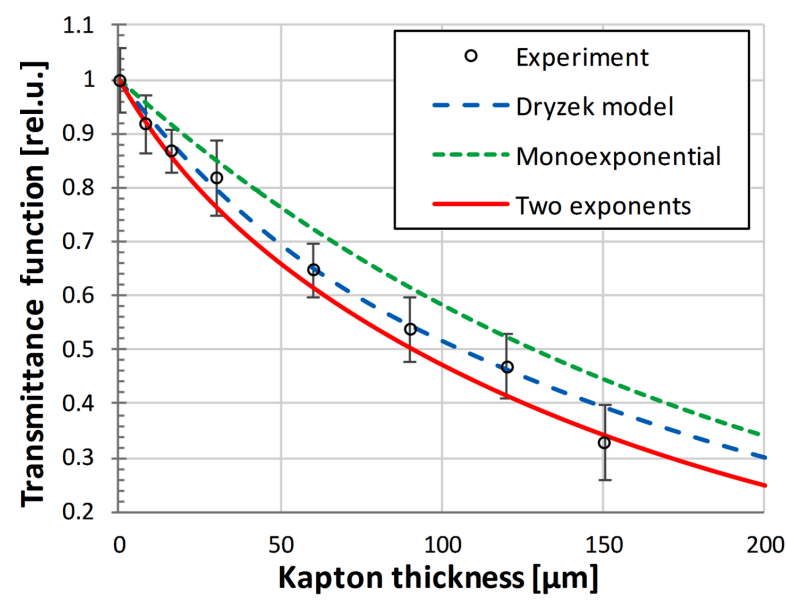

Fig. 6. Fraction of positrons annihilated in teflon samples in comparison with different transmittance functions: short-dashed line - Eq. (1), long-dashed line two-component Dryzek transmittance function $[1,4]$.

At small depths the difference between the Dryzek models (dashed lines) and our formula for kapton are non-important. It is within experimental uncertainties. However, at larger depths (>0.5 mm) it exceeds $100 \%$. For all low density materials the Dryzek implantation profiles decrease essentially slower than ours. Distinction between different approaches decreases with increasing density of material.

Figure 7 shows comparison between experimental data for Ni obtained by Hansen et al. [3] and two positron implantation profiles: calculated by Eq. (3) and obtained by differentiating the two-component the Dryzek function $I(z)$ given by Eq. (7). One can see that in the nearsurface region Dryzek implantation profile decreases with depth more slowly than our simulation (Eq. (3)) and the experimental data.

For the high density materials $\left(>11 \mathrm{~g} / \mathrm{cm}^{3}\right)$ distinction between the two approaches becomes practically insignificant.

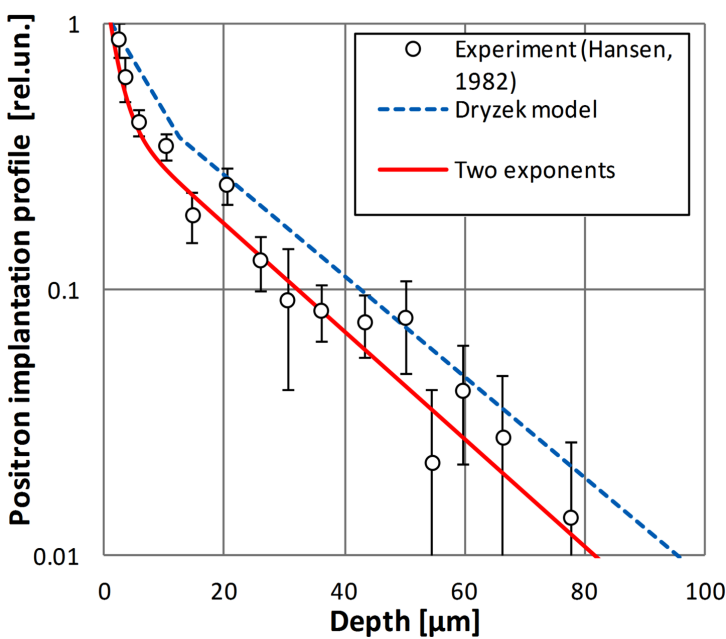

Fig. 7. Experimental ${ }^{22} \mathrm{Na} e^{+}$implantation profiles in Ni. Solid line - Eq. (3), dashed line is derived from the two-component Dryzek model [1]

\section{Conclusion}

Implantation profiles for positrons emitted by ${ }^{22} \mathrm{Na}$ source in a standard geometry of the positron lifetime measurements can be represented by the sum of two exponential functions. The proposed formula, Eq. (3), gives good approximation of the positron implantation profiles obtained by GEANT4 simulation for all investigated materials. The ratio between contributions of the long-range and short-range components for all materials remains the same. Thus, implantation profiles are determined only by two absorption coefficients, which depend on density and on average atomic number of the medium. For materials with atomic numbers from 6 to 82 , absorption coefficients can be calculated by means of the proposed formulae, Eqs. (4)-(5) with an accuracy of several percents.

\section{Acknowledgments}

This work was supported by the MEPhI Academic Excellence Project (Contract No. 02.a03.21.0005).

\section{References}

[1] J. Dryzek, D. Singleton, Nucl. Instrum. Methods Phys. Res. B 252, 197 (2006).

[2] A. Bisi, G. Gambarini, L. Zappa, Nuovo Cim. B 53, 428 (1979).

[3] H.E. Hansen, S. Linderoth, K. Petersen, Appl. Phys. A 29, 99 (1982).

[4] J. Dryzek, K. Siemek, J. Appl. Phys. 114, 074904 (2013).

[5] S. Jan, G. Santin, D. Strul, et al., Phys. Med. Biol.. 49, 4543 (2004).

[6] Xia Ning, Xingzhong Cao, Chong Li, Demin Li, Peng Zhang, Yihao Gong, Rui Xia, Baoyi Wang, Long Wei, Nucl. Instrum. Methods Phys. Res. B 397, 75 (2017). 